\title{
Diagnostic de la Gestion des Effluens Liquides Hospitaliers de la Region de Casablanca-Settat
}

\author{
Deblij Sanaa, \\ Laboratoire Biochimie, Environnement \& Agroalimentaire, \\ URAC 36, Faculté des Sciences et Techniques de Mohammedia \\ Bahlaouan Bouchaib, \\ Institut Supérieur des Professions Infirmières et des \\ Techniques de Santé de Casablanca \\ Boutaleb Nadia, \\ El Antri Said, \\ Laboratoire Biochimie, Environnement \& Agroalimentaire, \\ URAC 36, Faculté des Sciences et Techniques de Mohammedia
}

URL:http://dx.doi.org/10.19044/esj.2019.v15n6p171

\section{Résumé}

Les effluents liquides hospitaliers dans la région de Casablanca Settat sont marqués par l'absence de procédures de leur gestion. Ils sont évacués avec des volumes importants dans les réseaux collectifs d'assainissement, sans traitement préalable. Ces rejets contiennent des substances dangereuses chimiques et pathogènes ce qui constitue des menaces sérieuses de pollution et de détérioration de la santé des populations._Ce travail est une étude exploratoire sur les pratiques qui s'exercent en matière de gestion des rejets liquides dans les établissements hospitaliers de la région de CasablancaSettat. La méthodologie s'appuie sur 3 méthodes d'investigation à savoir ; (a) l'enquête par questionnaire auprès des personnels qui président et gèrent directement les effluents liquides hospitaliers via un questionnaire, (b) aussi l'estimation de la quantité des effluents déversés sur la base de la capacité litière fonctionnelle de tous les hôpitaux de la région; et (c) enfin sur l'analyse systématique et reproductible des articles marocains traitant la problématique des effluents hospitaliers. Parmi les résultats saillants de l'étude furent;(a) le diagnostic des principales lacunes et dysfonctionnements en termes de gestion des effluents au niveau de la région Casablanca-Settat ; (b) la quantité énorme des effluents liquides hospitaliers déversés sans aucun traitement préalable ;(c) la mauvaise qualité des effluents liquides hospitaliers au Maroc ; (d) Même typologie des effluents liquides tant au niveau national qu'au niveau marocain; Finalement, quelques recommandations furent 
formulées pour tracer les perspectives concourant vers une prise en charge durable de ces rejets.

Mots-clés: Effluents liquides, Casablanca-Settat, démarche qualité, milieu hospitalier, capacité litière fonctionnelle, management

\title{
Diagnosis of the Management of Hospital Liquids Discharges in the Region of Casablanca-Settat
}

\author{
Deblij Sanaa, \\ Laboratoire Biochimie, Environnement \& Agroalimentaire, \\ URAC 36, Faculté des Sciences et Techniques de Mohammedia \\ Bahlaouan Bouchaib, \\ Institut Supérieur des Professions Infirmières et des \\ Techniques de Santé de Casablanca \\ Boutaleb Nadia, \\ El Antri Said, \\ Laboratoire Biochimie, Environnement \& Agroalimentaire, \\ URAC 36, Faculté des Sciences et Techniques de Mohammedia
}

\begin{abstract}
Hospital liquid effluents in the Casablanca Settat region are marked by the lack of procedures for their management. They are evacuated with large volumes in collective sanitation networks, without prior treatment. These releases contain chemical and pathogenic hazardous substances which constitutes serious threats of pollution and deterioration of populations. This work investigates practices in the management of liquid waste in hospitals in the Casablanca-Settat region. Our methodology is based on 3 investigation methods namely; (a) the questionnaire survey of personnel who directly manage and manage hospital liquid effluents via a questionnaire; (b) also the estimation of the quantity of effluents discharged on the basis of the functional litter capacity of all hospitals the region ; and (c) finally, the systematic analysis of Moroccan articles dealing with the issue of hospital effluents. Among the outstanding results of this study were: (a) the identification of the main deficiencies in terms of effluent management in the Casablanca-Settat region; (b) the enormous quantity of hospital liquid effluents discharged without any prior treatment, (c) the poor quality of hospital liquid effluents in Morocco; (d) Same typology of liquid effluents at
\end{abstract}


both national and Moroccan levels; Finally, some recommendations were made to outline the prospects for sustainable management of these releases.

Keywords: Liquid effluents, Casablanca-Settat, quality approach, hospital environment, functional litter capacity, management

\section{Introduction}

Les rejets hospitaliers liquides menacent l'environnement beaucoup plus que les effluents urbains (Verlicchi et al., 2012). Ces rejets sont placés aujourd'hui au centre des préoccupations de l'ensemble des acteurs du système de santé et de ceux de la protection de l'environnement, car ils constituent une source potentielle de la transmission des infections et des maladies épidémiques (loi 28-00, 2006 ; OMS, 2010 ; Dahir n 456-11, 2010).

Ces effluents contiennent divers, polluants, substances toxiques et contaminants, tels que les produits pharmaceutiques, les radionucléides, les solvants et les désinfectants. Et sont généralement évacués dans les réseaux communaux urbains sans traitement préalable (Boillot, 2008 ; Ameziane et Benaabidate, 2012 ; Berrada et al., 2014, Dahri, 2015).

Par ailleurs, du point de vue écologique, de tels effluents, s'ils ne sont pas traités correctement, pour que leur qualité soit ramenée aux normes réglementaires, avant de les rejeter, ils peuvent dégrader l'environnement naturel et créer un déséquilibre biologique (Gautam et al., 2007).

En l'occurrence, l'assurance qualité au sein des hôpitaux s'avère être une priorité et s'appuie sur plusieurs normes et standards. Une assurance qui doit être appliquée à tous les domaines de l'hôpital et surtout le processus de la gestion de ces effluents.

Concernant la région de Casablanca-Settat, avec ses deux préfectures et ses sept provinces, c'est la région la plus peuplée du Royaume Marocain soit $20,3 \%$ de la population nationale (Haut-commissariat des plans, 2014) et celle qui jouit de la plus grande capacité litière privée et publique qui est de 5193 lits (Direction générale des collectivités, 2015).

En outre, la région jouit de la plateforme la plus importante dédiée à la gestion des effluents hospitaliers constituée par ses Comités de Lutte Contre les Infections Nosocomiales (CLIN). Un point fort qui va faciliter le diagnostic de la gestion et l'instauration de la démarche qualité.

Nonobstant, le processus de gestion des effluents hospitaliers ne sont soumis à aucune procédure ou protocole approprié pour la lutte contre les infections et la pollution au niveau de la plupart des pays au monde (OMS, 2010). Alors que plusieurs standards sont en vigueur (Chartier et al., 2014) et doivent être instaurés.

La présente étude à la fois descriptive, quantitative et exploratoire, consiste à faire un diagnostic de ce processus de gestion des effluents 
hospitaliers de la région de Casablanca-Settat afin de le confronter et comparer aux normes en vigueur.

La typologie et quantité des effluents liquides hospitaliers de la région milieu d'étude seront aussi identifiées afin d'expliquer les éventuels d'impact par la présence de contaminants de nature chimiques, physiques et microbiologiques. Et ce afin de proposer des solutions prometteuses aux lacunes soulevées.

\section{Matériel et Méthodes}

\section{Milieu d'étude}

Le milieu d'étude concerne toutes les provinces et préfectures de la région Casablanca - Settat (Figure 1). Une région qui se caractérise par un climat doux, semi-aride à aride en été, influencé par le vent océanique, pluvieux en hiver ; Les précipitations moyennes annuelles sont aux environs $347 \mathrm{~mm}$;

La région est caractérisée par la présence de grands bassins hydrauliques qui l'alimente: Oum Er Rbia avec ses cinq barrages, Côtier Atlantique Casa et Côtier Atlantique Safi-El Jadida ;

La population est estimée à $351 \mathrm{Habitants} / \mathrm{km}^{2}, 7$ fois supérieure à la moyenne nationale qui est de $47,21 \mathrm{hab} / \mathrm{Km}^{2}$;

La région est également marquée par une diversité géographique composé de la mer et son littoral, d'un relief et d'un Sahel (monographie de la région Casablanca-Settat, 2015 ; monographie agricole de la région Casablanca-Settat, 2018).

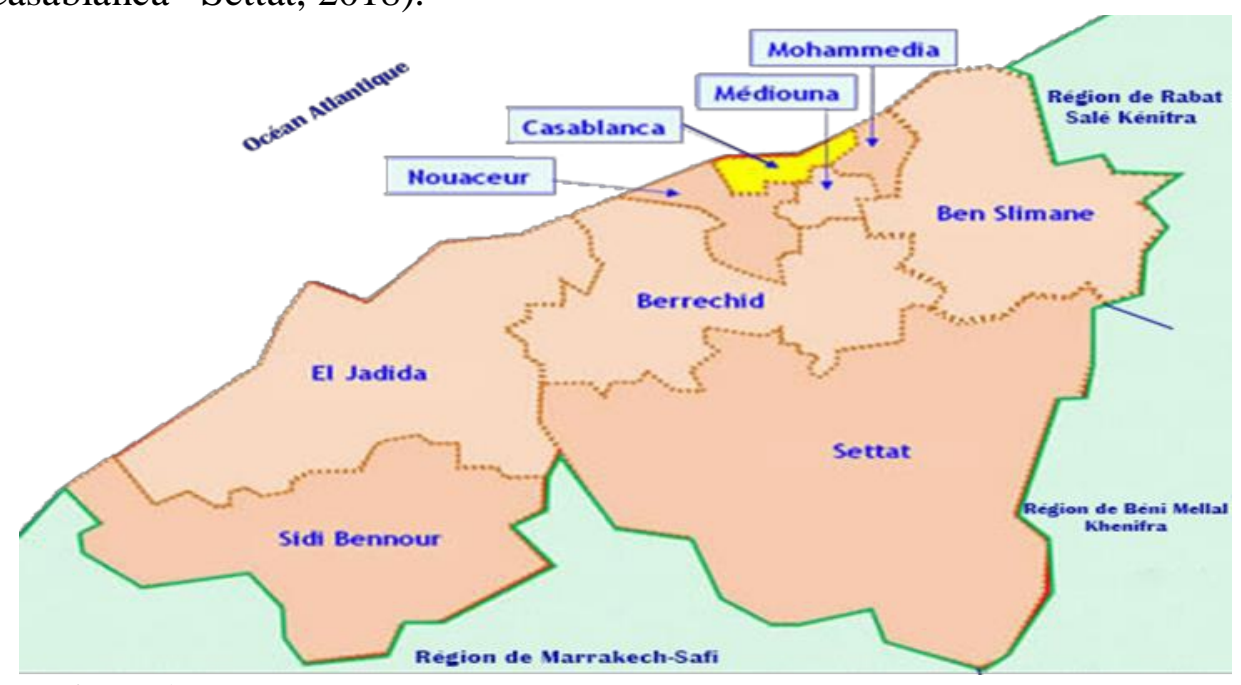

Figure 1 : Aperçu sur les provinces et préfectures de la région Casablanca - Settat

(Direction Régionale Casablanca-Settat, 2018) 
La région Casablanca- Settat dispose aussi d'une importante offre de soins hospitalière publique composée de 15 centres hospitaliers dont un CHR (Centre Hospitalier Régional) et un CHU (Centre Hospitalier Universitaire). En plus de 6 HP (Hôpitaux de Proximité) et 3 HS (Hôpitaux Spécialisés). Toutes les provinces et préfectures disposent d'un Centre Hospitalier Préfectoral (CHP) ou provincial à l'exception de la province de Benslimane qui ne dispose que d'un Hôpital de Proximité.

\section{L'enquête par questionnaire}

La plupart de ces hôpitaux disposent des CLIN (Comités de Lutte Contre l'Infection Nosocomiale) qui représentent des instances officielles responsables de l'hygiène hospitalière (décret n ${ }^{\circ} 991034$ du 6 décembre 1999), et sont aussi les premiers impliqués dans le processus de la gestion des effluents hospitaliers ;

$\mathrm{Au}$ niveau de la région Casablanca-Settat, ces comités contiennent dans la plupart des structures d'au moins cinq membres, qui ont été interpelés via un questionnaire et ce après avoir obtenu l'autorisation de collecte des données auprès de la directrice régionale de la santé Casablanca-Settat.

Le questionnaire était confectionné en trois parties : (a) une première partie portant sur des données générales d'identification des répondants ;(b) une deuxième partie qui traite le processus de la gestion des effluents au sein de la région (c) et une troisième partie traitant les suggestions des interviewés quant à l'amélioration du processus de la gestion .

Dans ce cadre, un échantillonnage exhaustif a été adopté et cela après avoir obtenu les consentements des répondants après avoir expliqué le but et l'orientation de l'étude. L'enquête a duré quatre mois et ce avec l'appui documentaire du responsable de l'offre de soins au sein de la région. Ainsi, l'échantillon total pris pour cette étude est 5 membres du CLIN pour chacun de $\mathrm{N}=24$ hôpitaux (120 répondants au questionnaire).

\section{Estimation de la quantité des effluents liquides générés}

Selon les normes de l'OMS (Chartier et al., 2014), le calcul de la quantité des liquides hospitaliers est reliée à la spécificité de l'hopital en question (hopital général ou spécialisé), à sa capacité litiére et son niveau d'intervention (hopital local, provincial ou préfectoral, régional ou un centre universitaire). Ainsi ,la production d'eaux usées dans les hôpitaux de niveaux secondaire et tertiaire est principalement mesuréetenant compte du taux d'hospitalisation (litre d'eaux usées générées par jour d'hospitalisation des patients) (Chartier et al., 2014). 
Les taux de génération des effluents liquides hospitaliers sont estimés donc pour les:

- Hôpitaux de taille moyenne : 300 à 5001 par patient et par jour ;

- Grands hôpitaux : 400 à 7001 par patient et par jour ;

- Hôpitaux universitaires : 500 à 9001 par patient et par jour.

\section{Analyse documentaire et reproductible des statistiques des études marocaines traitant le sujet des effluents hospitaliers}

14 articles marocains traitant la thématique des effluents ont été identifiés dans la littérature. Nous avons inclus uniquement les articles écrits en langue française ou anglaise et traitant les hôpitaux du Maroc.

Ces articles ont été exploités et interrogés à l'aide des mots clés tels que ; l'année et le milieu de la publication de l'étude ; les services hospitaliers milieux d'étude, la caractérisation des effluents étudiés, et les résultats obtenus.

En gros, la combinaison des trois méthodes à savoir l'enquête par questionnaire et l'analyse documentaire et l'estimation des quantités des effluents est faite afin de confronter, de renforcer les résultats obtenus des deux méthodes par la recherche de leur convergence et divergence.

\section{Résultats et discussions}

\section{Taux de participation}

Quatre comités parmi les 24 CLIN enquêtés n'étaient pas fonctionnels. Le questionnaire est distribué sur 100 membres, seulement 65 questionnaires sont récupérés. Le taux de réponse global était donc de $65 \%$.

\section{Qualification et fonctionnement du service}

Le tableau 1 présente les résultats de l'enquête par questionnaire concernant la qualification en termes d'expérience ont été traduits par le nombre d'années d'intervention en tant que membre du CLIN.

Tableau 1 : Nombre d'années d'intervention en tant que membre du CLIN

\begin{tabular}{|c|c|c|}
\hline Nombre d'années & Effectif & Fréquence \\
\hline$<1$ an & 2 & $3,08 \%$ \\
\hline$[1-3[$ & 3 & $4,62 \%$ \\
\hline$[3-5[$ & 18 & $27,69 \%$ \\
\hline$\geq 5$ & 42 & $64,61 \%$ \\
\hline
\end{tabular}

Beaucoup plus que la moitié des participants, soit $64,61 \%$ ont confirmé avoir passé plus de 5 années à intervenir en tant que membres du CLIN au sein de leurs services, alors que 27,69\% ont déclaré avoir une expérience de 3 à moins de 5 ans et moins de 3 ans en la matière. Une minorité par contre, à 
savoir 4,61\% des interviewés ont précisé n'avoir commencé à intervenir que depuis moins de 1 an.

Pour ce qui est des caractéristiques de fonctionnement (tableau 2), la majorité des répondants déclare à l'unanimité que leur travail au sein des comités est secondaire et non primaire et dépend des besoins des services.

Tableau 2 : les caractéristiques de fonctionnement selon les horaires de travail

\begin{tabular}{|c|c|c|}
\hline Horaire de travail adopté & Effectif & Fréquence \\
\hline Normal & 3 & $4,61 \%$ \\
\hline Système de garde & 5 & $7,69 \%$ \\
\hline En permanence & 5 & $7,69 \%$ \\
\hline En fonction des besoins des services & 52 & $80 \%$ \\
\hline
\end{tabular}

\section{Formation continue}

La formation continue est un outil pratiqué dans les structures par ce qu'elle apporte plusieurs avantages. En effet, elle contribue à l'accroissement de l'efficacité, la capacité d'accomplir les tâches demandées, la motivation. Les nouvelles compétences acquises suite à la formation continue par les employés améliorent la qualité du travail d'équipe, ainsi que son rendement et permet aussi de renfoncer la confiance de l'employé vis-à-vis de la structure à laquelle il dépend.

Il est donc utile de s'informer sur la participation ou non des présidents de CLIN à des formations continues sur la gestion des effluents liquides.

Pour la majorité des répondants, soit exactement $95 \%$, ils n'ont participé à aucune formation continue abordant la thématique de la gestion des effluents hospitaliers au sein de la région Casa-Settat.

\section{Système documentaire et réglementaire concernant la gestion des effluents de la région}

Le système documentaire et la traçabilité font partie intégrante de la démarche d'un travail de qualité. A cet effet, 77\% des répondants déclarent n'avoir jamais établi des rapports sur la gestion des effluents. Les résultats ont montré aussi en une unanimité l'existence d'une carence réglementaire nationale handicapante en matière du processus de gestion de ces effluents liquides hospitaliers.

En plus, la figure 2 illustre qu'une proportion importante de $61 \%$ déclare que les référentiels, les guides ou les fiches techniques de bonnes pratiques de la gestion des effluents existent dans la littérature mais ne sont pas appliqués. 


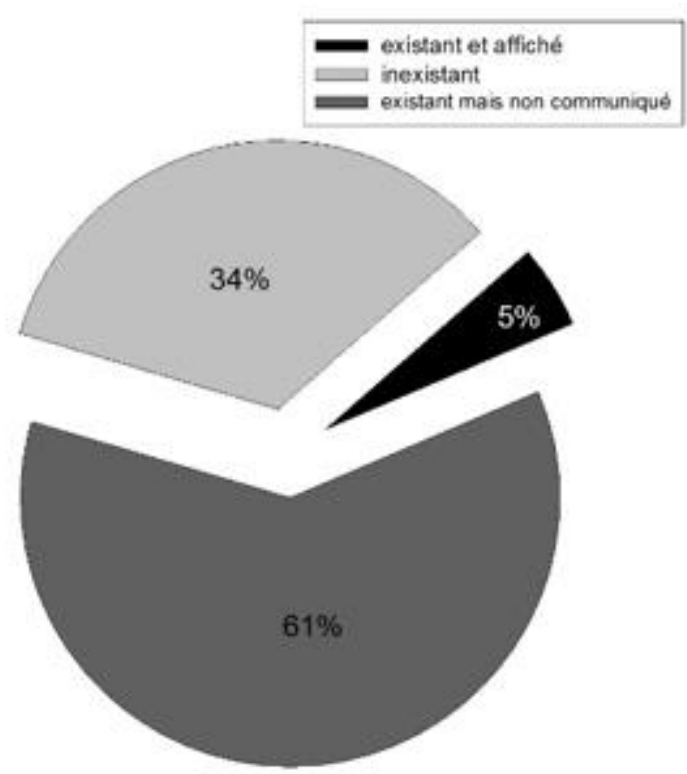

Figure 2 : Existence et communication de référentiels, de guides ou de fiches techniques de bonnes pratiques de gestion des effluents

\section{Quantité des effluents liquides générés}

Selon les données collectées depuis la Direction Régionale Casablanca-Settat réunies au niveau du tableau 3, la capacité litière des 24 hôpitaux de la région est estimée à 59\%.

Tableau 3 : la capacité litière des hôpitaux de la région Casablanca-Settat en 2018 (Direction régionale Casablanca-Settat, 2018)

\begin{tabular}{|c|c|c|}
\hline Préfecture / Province & Nom \& Type & $\begin{array}{c}\text { Capacité litière } \\
\text { fonctionnelle }\end{array}$ \\
\hline \multirow{2}{*}{ Ain Chok } & CHP Sekkat & 68 \\
\cline { 2 - 3 } & Centre National de léprologie & 10 \\
\hline $\begin{array}{c}\text { Ain Sebaa Hay } \\
\text { Mohammadi }\end{array}$ & CHP Mohammed V & 159 \\
\hline Ben M'sick & CHP Ben M'sick & 36 \\
\hline \multirow{2}{*}{ Casa Anfa } & CHR Moulay Youssef & 162 \\
\cline { 2 - 3 } & CHU & 1619 \\
\hline El Fida Mers Sultan & CHP Baouafi & 211 \\
\hline Hay Hassani & CHP Al Hassani & 52 \\
\hline Moulay Rachid & CHP Sidi Othmane & 148 \\
\hline \multirow{2}{*}{ Sidi Bernoussi } & CHP Al Mansour & 76 \\
\hline Mohammedia & HP Sidi Moumen (nouveau) & - \\
\hline Benslimane & CHP Moulay Abdellah & 118 \\
\hline \multirow{2}{*}{ Berrchid } & HP Benslimane & 32 \\
\cline { 2 - 3 } & CHP Berrchid & 45 \\
\hline
\end{tabular}




\begin{tabular}{|c|c|c|}
\hline \multirow{2}{*}{ El Jadida } & CHP Mohammed V & 333 \\
\cline { 2 - 3 } Médiouna & HP Azemmour & 40 \\
\hline \multirow{2}{*}{ Nouaceur } & HS de psychiatrie Arrazi & 118 \\
\cline { 2 - 3 } Settat & HP Médiouna (nouveau) & - \\
\hline \multirow{2}{*}{ Sidi Bennour } & CHP PH Moulay Hassane & 50 \\
\hline & HP Ben Ahmed & 258 \\
\hline \multirow{2}{*}{ Total Région } & CHP Sidi Bennour & 54 \\
\hline \multirow{2}{*}{ : CenR / 13 CHP / 3 HS / 6 HP } & $\mathbf{3 8 8 3}$ \\
\hline
\end{tabular}

CHR : Centre Hospitalier Régional ; CHP : Centre Hospitalier Préfectoral ; HS :Hôpitaux Spécialisés ; HP : Hôpitaux de Proximité

Selon les recommandations de l'OMS pour l'estimation de la quantité des effluents générée, décrites auparavant (Chartier et al.,2014), on déduit que pour:

- Les 12 hôpitaux de taille moyenne doivent consommer entre 300 à 500 litres par patient et par jour.

- Les 9 grands hôpitaux doivent avoir une consommation entre 400 à 700 litres par patient et par jour.

- Le seul hôpital universitaire, il doit consommer entre 500 à 900 litres par patient et par jour.

\section{Typologie des effluents hospitaliers}

Le point a été aussi mis sur les types d'effluents hospitaliers existants au sein de la région Casablanca-Settat. La majorité des interrogés (figure 3) citent les effluents chimiques, les métaux lourds, les rejets des désinfectants, des antiseptiques, des acides, les rejets médicamenteux, les rejets contenant des éléments pathogènes.

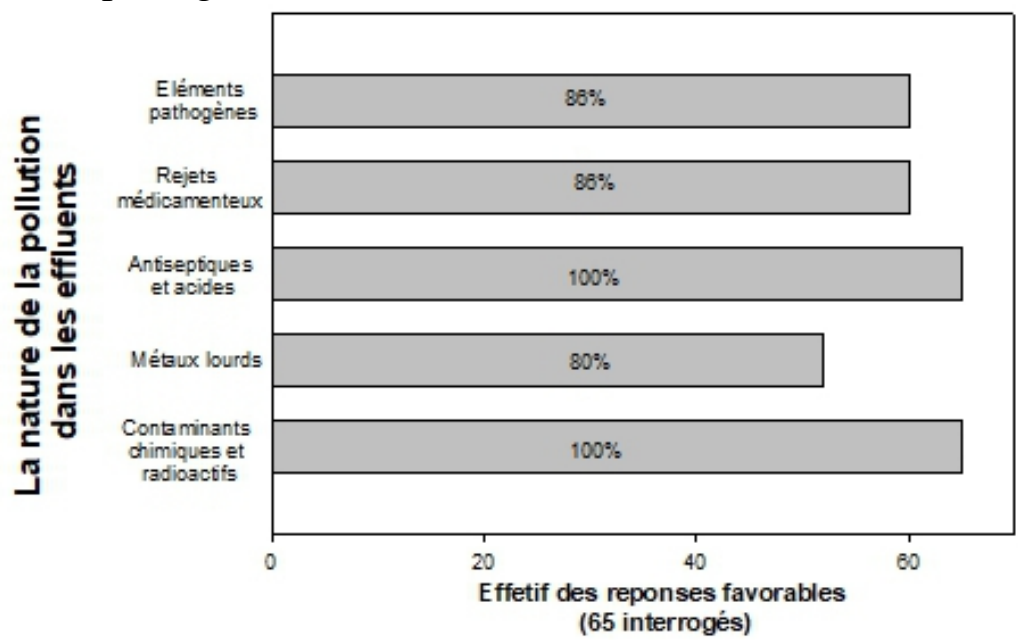

Figure 3: Les différents types de contaminants hospitaliers dans la région Casablanca-Settat 


\section{Gestion des effluents hospitaliers et étude de leur impact}

Le processus de la gestion de ces différents types d'effluents comprend plusieurs étapes. Et ce sont les membres du CLIN qui sont censés participer lors de toutes les étapes pour pouvoir veiller au respect des règles relatives aux pratiques de la gestion normative. La figure 4 montre que $49,62 \%$ de ces derniers n'interviennent dans aucune des étapes contre une petite minorité de 9.23\% qui déclarent avoir participé lors de l'étape du traitement.

En effet, $80 \%$ des répondants ont déclaré avoir conscience des risques vue la quantité énorme rejetée jugé par $76 \%$ et l'absence des stations de prétraitement dans tous les hôpitaux casablancais. Donc, les risques doivent être identifiés et évalués.

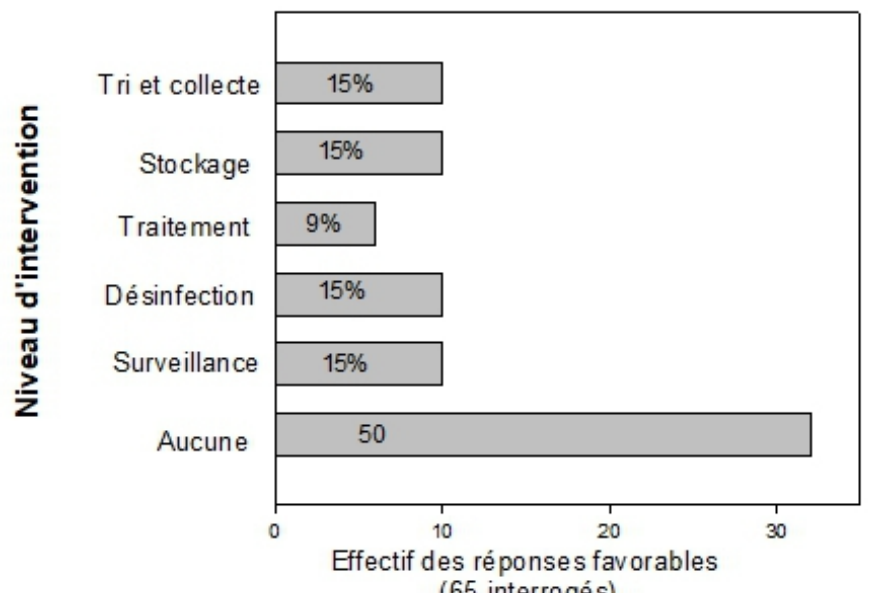

(65 interrogés)

Figure 4: Le niveau d'intervention dans le processus de la gestion des effluents hospitaliers

\section{Les risques générés par les effluents hospitaliers}

La figure 5 présente les résultats concernant et recensement et l'évaluation des risques.

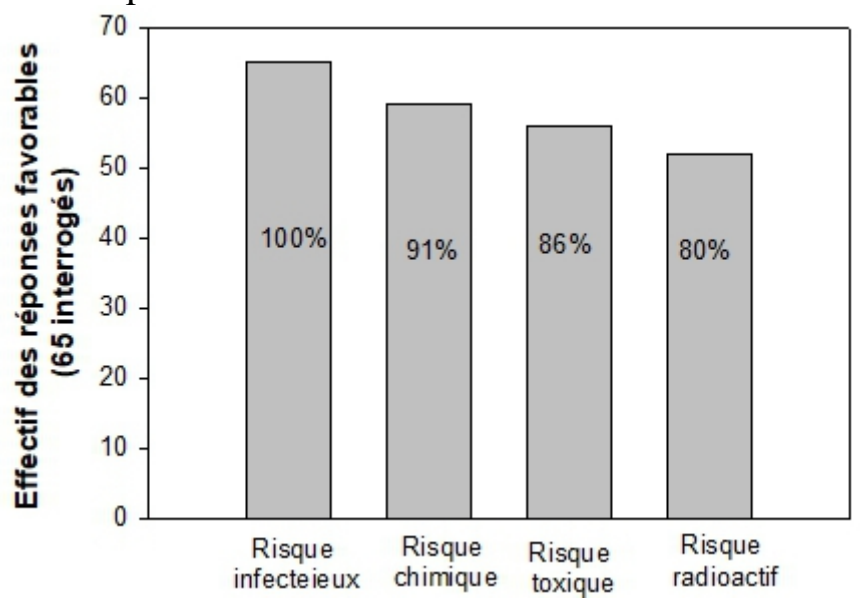

Figure 5: Les risques engendrés par les effluents hospitaliers 
On pourrait déduire que les répondants sont conscients des risques des effluents hospitaliers et sont unanimes sur le fait que ces effluents peuvent causer de multiples risques.

Causes de la mauvaise qualité des effluents hospitaliers au niveau de la région Casablanca-Settat

Tous les répondants au questionnaire ont été unanimes sur le fait que les effluents étaient de mauvaise qualité. Sur ce, une attention particulière doit être accordée à ses effluents et à leur impact sur l'environnement qui est dû à des causes illustrées dans la figure 6 .

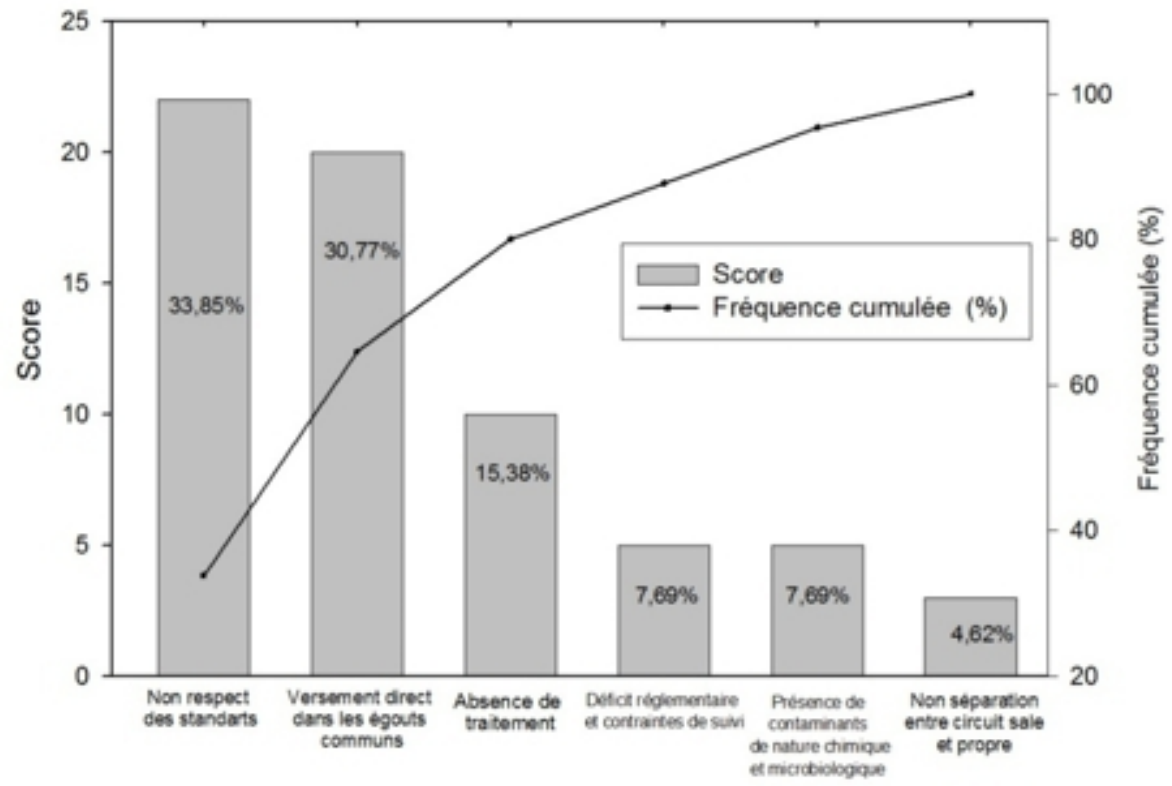

Figure 6: Diagramme de Pareto de la mauvaise qualité des effluents hospitaliers

D’après le graphique, il ressort que les causes "non-respect des standards " et "versement direct dans les égouts communs" et "absence de traitement" représentent $80 \%$ des causes de la mauvaise qualité des effluents hospitaliers de la région Casablanca-Settat. Ces causes doivent être analysées profondément pour définir des actions correctrices au niveau du processus de la gestion des effluents au niveau des hôpitaux de la région Casablanca-Settat.

\section{Analyse documentaire est reproductible des statistiques des études marocaines traitant le sujet des effluents hospitaliers}

L'analyse reproductible des résultats des études marocaines qui traitaient la même question de recherche de la gestion des effluents hospitaliers au Maroc, a fait ressortir seulement 14 articles marocains. Ces articles ont été inclus dans la présente analyse documentaire. 
Pour les années de ces publications de ces derniers, elles variaient entre 2012 et 2018.

Les critères d'inclusion dans l'étude étaient les suivants :

1. L'étude doit être menée dans une structure hospitalière dans une ville marocaine.

2. L'étude contient des données sur les échantillons (taille, période et lieu de collecte), les paramètres étudiés, et les principaux résultats obtenus.

Les 14 articles qui ont été inclus concernaient les villes marocaines suivantes : Rabat $(n=3)$, Sidi Kacem $(n=3)$, Fès $(n=3)$, Agadir $(n=1)$, Meknès $(n=2)$, Marrakech $(n=2)$ (tableau 4).

Tableau 4 : Les travaux réalisés sur les effluents hospitaliers au Maroc

\begin{tabular}{|c|c|c|}
\hline Zone d'étude & Intitulé de l'étude & Année \\
\hline \multirow[t]{3}{*}{ Rabat } & $\begin{array}{l}\text { The microbiological quality of water in Ibn Sina Hospital of Rabat } \\
\text { (Morocco) (Yagoubi et al., 2015). }\end{array}$ & 2015 \\
\hline & $\begin{array}{l}\text { Monthly physicochemical characterization of a Hospital effluent } \\
\text { according to technical and careactivities (Avicenna Rabat-Morocco) (El } \\
\text { Morhit } \text { et al., 2015). }\end{array}$ & 2015 \\
\hline & $\begin{array}{l}\text { Evaluation of biological and physicochemical risk of hospital liquid } \\
\text { waste in Morocco (Sarhane et al., 2017). }\end{array}$ & 2017 \\
\hline \multirow[t]{3}{*}{ Sidi Kacem } & $\begin{array}{l}\text { A principal component analysis (PCA) of hospital effluent pollution } \\
\text { levels (provincial hospital in Sidi Kacem Morocco) (Sadek et al., 2014a). }\end{array}$ & 2014 \\
\hline & $\begin{array}{l}\text { Contribution to the temporal assessment of releases provincial hospital } \\
\text { (sidi kacem, Morocco) (Sadek et al., 2014b). }\end{array}$ & 2014 \\
\hline & $\begin{array}{l}\text { The Bacterial Load of Hospital Discharges (Sidi Kacem, Morocco) } \\
\text { (Sadek et al., 2013). }\end{array}$ & 2013 \\
\hline \multirow[t]{3}{*}{ Fès } & $\begin{array}{l}\text { Recyclage des effluents du service d'hémodialyse de l'hôpital Al } \\
\text { Ghassani de la ville de Fès : caractérisation avant et après traitement } \\
\text { (Berrada, 2014) }\end{array}$ & 2014 \\
\hline & $\begin{array}{l}\text { Démarche environnementale : expérience d'un centre de dialyse (Dahri, } \\
\text { 2015) }\end{array}$ & 2015 \\
\hline & $\begin{array}{l}\text { Characterization of the chemical and bacteriological risks of the effluents } \\
\text { from some services of the Hassan II Hospital Center in Fez (El } \\
\text { Mountassiret al., 2017). }\end{array}$ & 2017 \\
\hline \multirow[t]{2}{*}{ Meknès } & $\begin{array}{l}\text { Caractérisation microbiologique des effluents de l'hôpital Mohamed V } \\
\text { de Meknès et étude de leur impact sur l'environnement (Ameziane et } \\
\text { Benaabidate, 2012) }\end{array}$ & 2012 \\
\hline & $\begin{array}{l}\text { Estimate of the metallic Contamination of the urban effluents By the } \\
\text { effluents of the Mohamed V Hospital of Meknes (Ameziane et } \\
\text { Benaabidate, 2014) }\end{array}$ & 2014 \\
\hline \multirow[t]{2}{*}{ Marrakech } & $\begin{array}{l}\text { A survey of wastewaters generated by a hospital in Marrakech city and } \\
\text { their characterization (El Ogri et al., 2016). }\end{array}$ & 2016 \\
\hline & $\begin{array}{l}\text { Application d'une méthode d'étude quantitative et qualitative des rejets } \\
\text { liquides hospitaliers au niveau de la Région de Marrakech Tensift El } \\
\text { Haouz (Qadouri et al., 2016). }\end{array}$ & 2016 \\
\hline Agadir & $\begin{array}{l}\text { Monitoring of the Microbiological Quality of Dialysate and Treated } \\
\text { Water (Asserraji, 2014) }\end{array}$ & 2014 \\
\hline
\end{tabular}




\section{Services les plus producteurs des effluents de mauvaise qualité}

L'ensemble des résultats des articles analysés montrent que les rejets liquides hospitaliers des différents hôpitaux marocains sujets d'études des articles précités étaient de mauvaise qualité et deviennent dangereux surtout lorsqu'ils proviennent de certains services particuliers comme le service d'hémodialyse, de la radiologie et le laboratoire (tableau 5).En plus, la caractérisation physico-chimique, bactériologique et microbiologique de ses effluents a montré l'acidité légère des $\mathrm{pH}$, l'augmentation de la minéralisation, de la température, de la salinité, de la conductivité et un accroissement très significatif des valeurs de la DCO et de la DBO5. Il a été démontréune faible biodégradabilité due à la nature chimique des rejets hospitaliers avec une charge bactériologique importante. Il a été noté aussi la présence de germes multirésistants et virulents de nature émergente tels que Pseudomonas aeroginosa ; Et la présence des oligo-éléments tels que le mercure (les concentrations de mercure sont dix fois supérieures à la valeur seuil 0,05 mg / 1), le cuivre et le chlore. En fait,les auteurs ont trouvé que ces effluents ont des effets néfastes sur la vie humaine, les animaux, l'environnement et les écosystèmes en général.

Tableau 5: Services les plus producteurs des effluents hospitaliers

\begin{tabular}{|c|c|}
\hline $\begin{array}{c}\text { Les services les plus producteurs des } \\
\text { effluents liquides }\end{array}$ & Effectif \\
\hline Tous les services & 6 \\
\hline Hémodialyse & 5 \\
\hline Radiologie & 3 \\
\hline Laboratoire & 3 \\
\hline Pharmacie & 3 \\
\hline Maternité & 4 \\
\hline Médecine et chirurgie & 3 \\
\hline Urgences & 4 \\
\hline
\end{tabular}

La caractérisation des effluents des services suscités a montré donc que les effluents de tous ses services sont de mauvaise qualité. En l'occurrence, ceux provenant de l'hémodialyse, la maternité, la radiologie, la pharmacie, le laboratoire et les urgences sont aussi des services producteurs.

L'enquête par questionnaire a rajouté la chambre mortuaire, l'oncologie et le bloc opératoire comme service de grande production des effluents.

\section{Qualité des effluents hospitaliers au niveau des hôpitaux marocains}

Toutes les études compulsées corroborent que les eaux usées des hôpitaux sont généralement évacuées dans les réseaux urbains sans aucun traitement. Qu'elles sont de mauvaise qualité et ils recommandent qu'ils soient préalablement bien traités. L'ensemble des résultats montre également que ces 
eaux présentent des signes de dégradation importante, puisque la majorité des paramètres étudiés dépassent les normes préconisées par l'OMS et les normes Marocaines (Bulletin officiel, 2002).

\section{Réduction de l'impact des effluents hospitaliers}

La figure 7 présente les solutions proposées par les interrogés afin de réduire l'impact sur l'environnement.
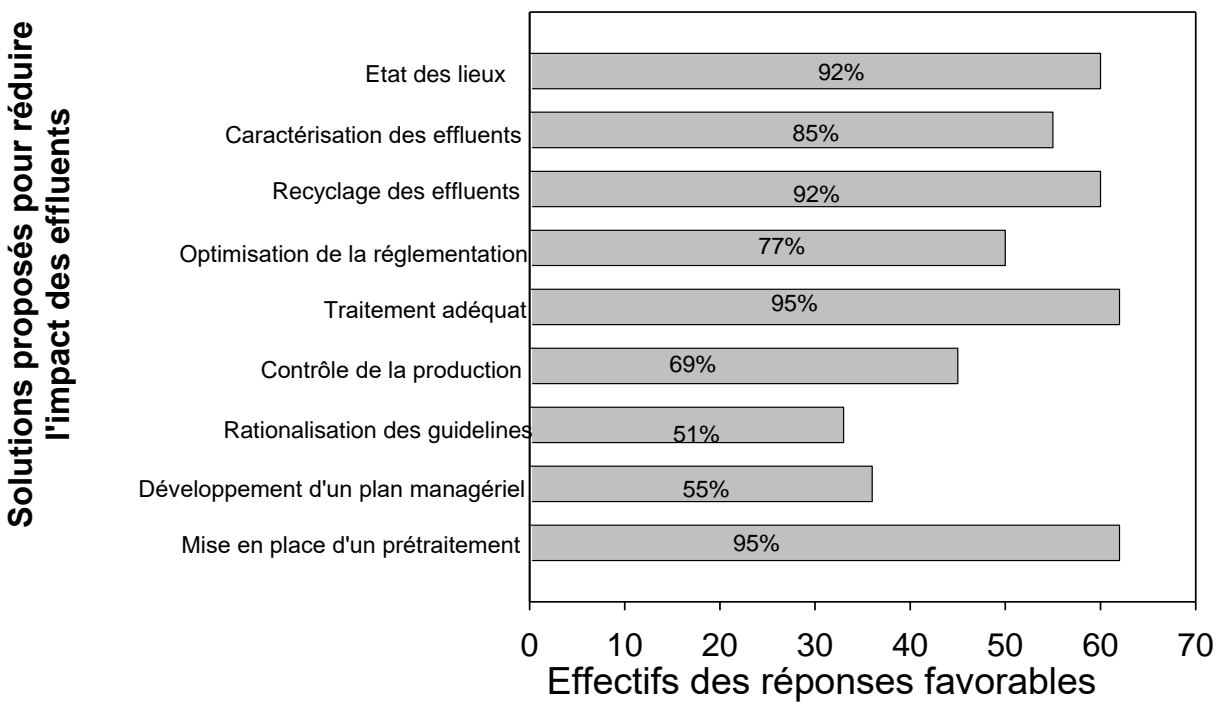

(65 interrogés)

Figure 7: Propositions des répondants pour réduire l'impact des effluents

Les apports des participants en terme de propositions pour améliorer le processus de gestion et réduire l'impact des effluents furent très pertinentes et portèrent notamment sur : (a) le traitement adéquat des effluents ; (b) la mise en place d'un prétraitement de ces derniers ; (c) la mise en place d'un état de lieux ; (d)le recyclage des effluents et leur traitement adéquat ;(e) leur caractérisation et le contrôle de leur production ; (f) l'optimisation de la carence réglementaire et la rationalisation des guidelines.

\section{Discussion}

La présente enquête est nécessaire vue qu'elle diagnostique les pratiques de gestion des effluents hospitaliers, qualifiés de dangereux vu qu'ils contiennent des substances chimiques très toxiques et des contaminants biologiques. L'étude concerne une région qualifiée de plus polluée au Maroc. Ce diagnostic permettra de soulever les écarts et les lacunes pour des fins d'amélioration. 
Les gestionnaires de ces dits effluents au niveau de la région CasaSettat n'ont bénéficié que de peu formation continue en la matière. Les résultats de l'étude ont révélé aussi que $80 \%$ des membres ne s'y engagent pas de manière principale dans leur temps d'activité normale, mais juste secondairement selon les besoins des services hospitaliers.

Aussi les résultats de l'étude ont montré qu'au niveau de la région Casa-Settat, il existe des hôpitaux provinciaux ou préfectoraux sans CLIN fonctionnels ou que ceux-ci n'établissent pas de rapports périodiques. Résultat que corrobore le rapport de la cour des comptes de l'an 2016 qui a constaté que le CLIN chargé de prévenir les risques d'infections et de propagation de maladies est inexistant au niveau de du centre hospitalier préfectoral de Hay Hassani en dépit du fait que des notes de services prévoyant leur création ont été diffusées depuis février 2012 et en plus qu' aucun PV y concernant n'a été communiqué pour décider sur la fréquence de leurs réunions et les domaines de leurs concertations.

En plus, la présente étude a mis le point aussi sur les différents types d'effluents au sein de la région Casa-Settat. La majorité des répondants citent de manière unanime les effluents chimiques comme le glutaraldéhyde, et l'acide acétique et d'autres, les métaux lourds comme l'argent métallique et les sels d'argent et d'autres, les rejets des désinfectants, des antiseptiques, des acides, les rejets médicamenteux, les rejets contenant des éléments pathogènes comme le sang, les urines et d'autres.

Ces résultats sont proches de ceux des travaux effectués respectivement par Qadouri et al.,(2016) au niveau de la région de Marrakech Tensift El Haouz et le guide national de la gestion des déchets des établissements de soins élaboré par la direction des hôpitaux et des soins ambulatoires marocains.

Par ailleurs ; environ50 \% des enquêtés n'interviennent dans aucun niveau du processus de la gestion des effluents hospitaliers et aucune analyse physico-chimique n'a jamais été effectué sur les effluents des hôpitaux de la région Casa-Settat. En dépit du fait que $80 \%$ des répondants sont conscients des risques de ces derniers vue l'énorme quantité jugés par $76 \%$ des effluents versés directement sans prétraitement dans les égouts communs. Résultats qui concordent avec les chiffres de la quantification de ces derniers au sein de la région et de plusieurs enquêtes au niveau national et international (l'OMS, 2002 ; Qadouri et al., 2016 ; Dahri,2005).

Il est donc clair que les eaux au niveau de la région Casablanca-Settat sont menacées par des problèmes de grandes quantités et mauvaises qualités des effluents hospitaliers. Sachant bien que la production journalière des eaux 
usées au Grand Casa est estimé à $80 \mathrm{~L} / \mathrm{j}$ par habitant en contrepartie nos hôpitaux de la région génèrent entre 400 à $900 \mathrm{l} / \mathrm{j}$ par patient. Ce qui est très énorme.

Subséquemment, on peut dire que les hôpitaux casablancais génèrent quatre fois plus que les ménages des substances très dangereuses et les déversent dans deux fleuves qui sont, l'oued Oum Er Rbia et l'oued Bou Reg Reg. Malheureusement, la qualité de l'eau de la ville de Casablanca est très reliée à la qualité des flux de ces deux fleuves (Zidane et al, 2010).

Par rapport à un autre volet important et en relation avec l'environnement les résultats de l'étude ont montré qu'aucun système de traitement des effluents n'est de vigueur au niveau des hôpitaux en question, et surtout au niveau des services qui produisent beaucoup d'effluents et qui ont été évoqués dans la présente étude selon un ordre croissant du plus grand producteur au plus petit producteur à savoir le service d'oncologie, la chambre mortuaire, le laboratoire, le service de radiologie , d'hémodialyse, le bloc opératoire et la pharmacie....Ces résultats ont été affirmés par tous les participants, et confirmés aussi bien par l'analyse documentaire.

Nonobstant, le guide national de la gestion des déchets recommande la nécessité d'un prétraitement des effluents enproposant de petites stations d'épuration pour les grands hôpitaux ou des prétraitements spéciaux pour traiter les effluents des services connus par leurs rejets dangereux notamment l'oncologie, les laboratoires, la dialyse et autres.

Dans le même cadre des idées et En Italie, Espagne, Ukranie et Allemagne le prétraitement des effluents hospitaliers n'est pas obligatoire localement par contre l'OMS le préconise en parraléle avec la désinfection au chlore quand la masse de la matière organique est inférieure à $10 \mathrm{mg} / 1$. Pour la France la désinfection est recommandée par les lois communautaires, surtout lors des maladies infectieuses (Carrero et al.,2016).

En Iran, les hôpitaux sont obligés deprétraiter leurs eaux usées par eux même. Aussi, dans ces hôpitaux, les rejets ne sont pas déversés dans les réseaux communaux. Puisque, chaque hôpital gère ses rejets en ayant recours à un département de traitement qui lui est affilié et qui les réutilise (Mesdaghiniav et al., 2009) ; Surtout que dernièrement, ces effluents ont pu suscité l'intérêt de plusieurs chercheurs qui se sont alarmés concernant leurs risques chimiques, radiologiques et cancérogénes (Déblonde, 2013; Catastini et al, 2008).

Constat confirmé et par l'enquête par questionnaire qui a étalé les risques pouvant être engendrés et qui sont multiples et diversifiés et par l'ensemble des articles marocains analysés dans le cadre de cette étude. Dans 
un autre volet et sachant bien que la démarche qualité étant toujours maintenue par la documentation et l'existence des plans de gestion, les résultats de l'étude ont démontré que $95 \%$ des participants ne possèdent pas des guides, référentiels ou fiches techniques de bonnes pratiques. D’un autre côté, les observations au niveau de ces hôpitaux n'ont pu déceler l'existence d'une seule fiche technique sur la gestion des effluents liquides au niveau des hôpitaux de la région.

Concernant la dimension réglementaire, elle est d'une grande importance Toutefois, et à l'échelle nationale, cet aspect reste marqué par une déficience et un vide ce qui a été rapporté par tous les répondants. Et ce n'est qu'en 2006, que la première loi relative à la gestion des déchets solides en général et les déchets médicaux en particulier a vu le jour. Toutes ces lois n'évoquent pas la gestion des effluents hospitaliers.

Concernant ce volet réglementaire en Algérie, la gestion et le traitement des eaux usées et l'exigence de la protection de l'environnement sont devenues une priorité politique et en 2009 un décret est publié explicitant les modalités d'octroi de l'autorisation de déversement des eaux usées dans un réseau public d'assainissement (Kouidar et al., 2015)

Donc, il est impératif de repenser la gestion des effluents hospitaliers et re-enquêtera situation épidémiologique au niveau de la région Casa-Settat en la reliant à la pollution et la contamination par les effluents hospitaliers qui sont de mauvaise qualité.

\section{Conclusion}

La présente étude s'est accordée comme but de diagnostiquer la gestion des effluents liquides par les établissements de santé de la région de Casablanca-Settat. Les résultats obtenus ont montré que ces derniers sont peu mobilisés concernant la problématique des effluents hospitaliers qui sont déversés sans aucun traitement préalable et sans respect des règles relatives aux pratiques de gestion normatives en vigueur.

Cette étude représente donc une étape préliminaire dans la constitution d'une base de données pour des études ultérieures sur la gestion des effluents au niveau de la région.

Afin de pallier aux risques potentiels de cette mauvaise gestion des effluents soulevés tout au long de l'étude en l'occurrence l'inadéquation architecturale, l'absence d'un système de prétraitement, la carence réglementaire et l'absence d'un système documentaire, des recommandations ont été formulées aux suggestions des participants à l'étude en l'occurence : 
- L'instauration d'un plan de la gestion des effluents et clarification des responsabilités

- La caractérisation physico-chimique, bactériologique et toxicologique des effluents liquides issus des activités de soins et relier cette caractérisation à la situation épidémiologique au sein de la région surtout au niveau des hôpitaux de Sidi-Bennour, El Jadida et Grand Casa dans des études futures.

- La mise en place d'un prétraitement qui peut s'agir de petites stations d'épuration au sein des services à risque et des services grands producteurs.

- La prévention de l'augmentation de la production et de la nocivité de ces effluents.

- L'élaboration au niveau stratégique d'un arsenal réglementaire afin d'appuyer les pratiques de gestion des effluents hospitaliers au niveau de la région Casa-Settat

- Le recyclage des effluents hospitaliers des services qui produisent beaucoup plus de rejets liquides spécifiques aux activités soins.

\section{Remerciement}

Nos remerciements à la Direction Régionale de la Santé de Casablanca-Settat.

\section{References:}

1. Ameziane,N.Benaabidate, L.(2014). Estimate of the metallic Contamination of the urban effluents By the effluents of the Mohamed V Hospital of Meknes.EuropeanScientific Journal. 10 (3). pp70-78.

2. Ameziane, N., Benaabidate, L. (2012). Caractérisation microbiologique des effluents de l'hôpital Mohamed V de Meknès et étude de leur impact sur l'environnement. Nature et technologie. 10 (janvier).pp31-38.

3. Asserraji, M., Maoujoud, A.,Belarbi, M. Elfarouki,R.(2014).Monitoring of the MicrobiologicalQuality of Dialysate and Treated Water. Saudi Journal of Kidney Diseases and Transplantation. 25(1). pp91-95

4. Berrada, S., Squalli, F.Z., Squalli, H.T., Hannin, M., El Oualti, A., El OualiLalami A. (2014). Recyclage des effluents du service d'hémodialyse de l'hôpital Al Ghassani de la ville de Fès : caractérisation avant et après traitement. J. Mater. Environ. Sci. 5 (S1) (2014). pp 2265-2277

5. Boillot, C. (2008).Évaluation des risques écotoxicologiques liés aux rejets d'effluents hospitaliers dans les milieux aquatiques: 
Contribution à l'amélioration de la phase « caractérisation des effets. Institut National des Sciences Appliquées de Lyon. France. .299 pages

6. Carraro, E., Bonetta, Si. Bertino, C. Lorenzi , Sa. Gilli, G.(2016).Hospital effluents management: Chemical, physical, microbiological risks and legislation in different countries. Journal of Environmental Management.2016.P193-194.

7. Catastini,C., Mullot,J.U., Boukari,S., Mazellier,P., Levi,Y., Cerv antes P. et Ormsby J.N.Identification de molécules anticancéreuses dans les effluents hospitaliers.Journal Européen d'hydrologie. 39( 2), 2008. pp171 - 180.

8. Dahir ${ }^{\circ} 1-06-153$ du 30 chaoual 1427 (22 novembre 2006) portant promulgation de la loi $\mathrm{n}^{\circ} 28-00$ relative à la gestion des déchets et à leur élimination. (86Articles) - B.O. $\mathrm{N}^{\circ} 5480$ DU 15 Kaada 1427 (7/12/2006). 27 pages.

9. Dahir $\mathrm{n}^{\circ} 456-11$ du 2 Rajeb 1431 (6 juillet 2010) portant sur le Règlement Intérieur des Hôpitaux. Bulletin Officiel n ${ }^{\circ} 5926$ du 12 Rabii II 1432 (17 Mars 2011). Ministère de la Santé, Royaume du Maroc.https://pharmacie.ma/uploads/pdfs/reglement_int_des_hop.pdf (Consulté le 26 novembre 2018)

10. Dahri,S.(2015). Démarche environnementale : expérience d'un centre de dialyse. Thèse en médecine. Faculté de médecine et de pharmacie de Fés. Maroc. 65 pages

11. Deblonde, T.(2013). Evaluation des risques sanitaires de la consommation d'eaux potentiellement chargées en résidus de médicaments anticancéreux. Thèse en medecine. Université de Lorraine, 2013. . 185 pages.

12. Direction Générale des Collectivités Locales. (2015). Monographie de la région Casa-Settat. Royaume Du Maroc Ministère de l'Intérieur. http://casablanca.ma/RS/Doc/monographie-region-2015.pdf.

(Consulté le 26 novembre 2018)

13. El-Ogri, F., Ouazzani,N., Boraâm, Mandi L. (2016).A survey of wastewaters generated by a hospital in Marrakech city and their characterization. Desalination and Water Treatment.57 (36). pp 1706117074.

14. El Mountassir, R., Bennani, B., Merzouki, H., Benjelloun Touimi, G., Boumchita, S., Benjelloun, Y., Lahrichi, A. (2017).Characterization of the chemical and bacteriologicalrisks of the effluents fromsome services of the Hassan II Hospital Center in Fez. Journal of Materials and Environmental Sciences 8(7). pp2288-2295.

15. El Morhit, M., Yagoubi,M., Belmakki, A., Zouhdi, M.(2015) Monthly physicochemical characterization of a Hospital effluent according to 
technical and care Cctivities (avicenna rabat-morocco). World journal of pharmacy and pharmaceutical sciences. 4(4). pp247-267.

16. Gautam, A. K., Kumar, S., Sabumon, P.C. (2007) Preliminary study of physico-chemical treatment options for hospital wastewater.Journal of Environmental Management. 83(3). pp298-306.

17. Haut-Commissariat au Plan. (2014). Recensement générale de la population et de l'habitat. Maroc. http://rgphentableaux.hcp.ma/ (Consulté le 26 novembre 2018)

18. Kouider Moussaoui, A. (2015).Traitement des déchets (solides et liquides) des hôpitaux. Cas : l'hôpital Makour Hammou d'Ain Defla. PFE de master en génie des procédés. Algérie.

19. Lebrun, F. M.(2000). Les rejets liquides des établissements de santé, Caractérisation à la source et impact sur l'environnement marin côtier. Centre Hospitalier du Havre - CLIN - Club Environnement.Paris.http://greeqs.free.fr/siteeqs/eau/textes/nonregl/A UT-01-00A.pdf. (Consulté le 26 novembre 2018)

20. Mesdaghinia, AR . Naddafi, K. Nabizadeh, R . Saeedi, R. Zamanzadeh, M.(2009) Wastewater Characteristics and Appropriate Method for Wastewater Management in the Hospitals.Iranian .J Publ Health, Vol. 38, No.1, 2009, pp.34-40.p34.

21. Ministère de la santé, stratégie sectorielle de la santé 2012-2016. Maroc.

2012(Mars).file:///C:/Users/HP/Downloads/secteur\%20sant\%C3\%A9 $\% 20$ (2).pdf (Consulté le 26 novembre 2018)

22. Organisation Mondiale de la Santé, Comité régional de la Méditerranée orientale. (2010). Lutte contre les infections et soins de santé : nécessité d'une action de collaboration. Comité régional de l’EM/RC57/6 Méditerranée orientale. 